
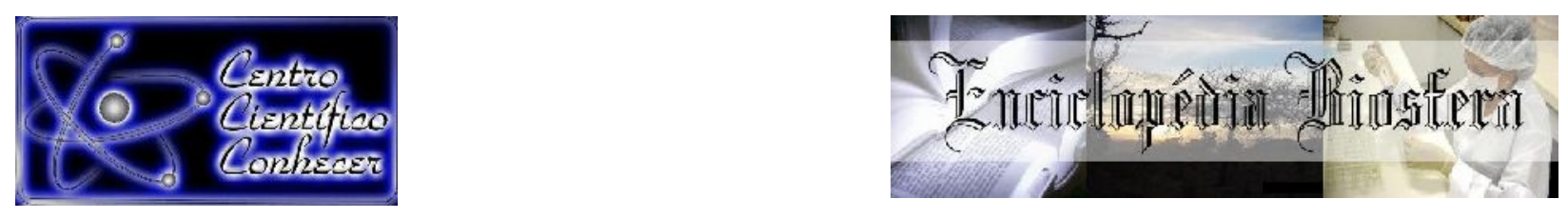

\title{
ASPECTOS ECONÔMICOS E SANITÁRIOS SOBRE A CONDENAÇÃO TOTAL DE CARCAÇAS BOVINAS POR CISTICERCOSE NO ESTADO DE MATO GROSSO DO SUL
}

\author{
Weyber Ferreira de Souza', Guilherme Augusto de Campos', Diego Pierotti \\ Procópio $^{2}$ \\ ${ }^{1}$ Graduando em Medicina Veterinária pela Universidade Federal de Mato Grosso, \\ Cuiabá - MT, Brasil. (weyberfs@gmail.com). \\ ${ }^{2}$ Orientador, Professor da Faculdade de Agronomia e Zootecnia na Universidade \\ Federal de Mato Grosso - Campus Cuiabá. Cuiabá-MT, Brasil.
}

Recebido em: 22/09/2018 - Aprovado em: 23/11/2018 - Publicado em: 03/12/2018 DOI: 10.18677/EnciBio_2018B11

\begin{abstract}
RESUMO
Objetivou-se nesse estudo determinar a perda econômica das condenações totais de carcaças bovinas ocasionadas pela cisticercose para o estado de Mato Grosso do Sul no período de 2013 a 2017. A região Centro-Oeste realizou o abate de 54,43 milhões de bovinos no período de 2013 a 2017, com destaque para os estados de Mato Grosso (23,26 milhões) e Mato Grosso do Sul (17,10 milhões). Com o objetivo de garantir a segurança dos alimentos, no processamento industrial, as carcaças são fiscalizadas pelo Sistema de Inspeção Federal (SIF) e, caso apresentem algum problema patogênico ou não patogênico, pode haver a condenação total ou parcial. Dentre as causas de condenação de carcaças bovinas, tem-se a cisticercose, que é uma doença de caráter zoonótica e parasitária, caracterizada pela presença das formas larvais das Taenia saginata. No estado de Mato Grosso do Sul, entre os anos de 2013 a 2017, foram registrados 15,7 mil casos de condenações totais de carcaças bovinas em virtude da cisticercose e a perda econômica estimada foi de $\mathrm{R} \$ 34,12$ milhões. Sendo assim, recomenda-se a adoção de ações que visem à interrupção do ciclo da cisticercose bovina, como a conscientização dos funcionários de propriedades rurais quanto à importância da doença e sobre a realização do manejo ambiental e do tratamento antiparasitário. Bem como a ampliação do serviço de inspeção sobre a etapa de abate e processamento de carne bovina comercializada no mercado nacional.
\end{abstract}

PALAVRAS-CHAVE: Carne Bovina, Inspeção, Perda Econômica, Saúde Pública, Taenia Saginata

\section{ECONOMIC AND SANITARY ASPECTS ON THE TOTAL CONDEMNATION OF BOVINE CARCASSES BY CYSTICERCOSE IN THE STATE OF MATO GROSSO DO SUL}

\footnotetext{
ABSTRACT

The objective of this study was to determine the economic loss of the total condemnations of bovine carcasses caused by cysticercosis to the state of Mato Grosso do Sul in the period from 2013 to 2017. The Central-West region slaughtered 54,43 million cattle in the period from 2013 to 2017, featured with the states of Mato ENCICLOPÉDIA BIOSFERA, Centro Científico Conhecer - Goiânia, v.15 n.28; p.124 2018
} 
Grosso (23,26 million) and Mato Grosso do Sul (17,10 million). With the objective ensure to food safety, in industrial processing, the carcasses are inspected by the Federal Inspection System (FIS) and, if they present any pathogenic or nonpathogenic problem, there may be total or partial condemnation. Among the causes of condemnation of bovine carcasses is cysticercosis, which is a zoonotic and parasitic disease, characterized by the presence of larval forms of Taenia saginata. In the state of Mato Grosso do Sul, between the years of 2013 and 2017, there were 15,70 thousand cases of total condemnation of bovine carcasses due to cysticercosis and the economic loss calculate was $R \$ 34,12$ million. Therefore, it is recommended the adoption of actions that aim to interrupt the cycle of bovine cysticercosis, such as the awareness of rural property employees regarding the importance of the disease and the realization of environmental management and antiparasitic treatment. As well as the extension of the inspection service on the slaughtering and processing of beef sold in the domestic market.

KEYWORDS: Beef, Inspection, Public Health, Economic Loss, Taenia Saginata

\section{INTRODUÇÃO}

Entre 2013 e 2017, o Brasil realizou 120,5 milhões de abates de bovinos em frigoríficos sob fiscalização do Serviço de Inspeção Federal (SIF). O estado do Mato Grosso do Sul, se figura como o segundo maior produtor, em termos de abates, tendo realizado 17,1 milhões de abates nesse período, perfazendo $14,20 \%$ dos abates total nacional (MAPA, 2018).

Os produtos de origem animal possuem características que podem favorecer o desenvolvimento de patógenos. Dessa forma, caso a carne esteja contaminada e seja consumida pela população, representa um problema de saúde pública (PORTILHO, 2016).

Com o intuito de garantir a segurança dos alimentos e a qualidade da carne bovina que é comercializada, nas indústrias de processamento são realizadas inspeções sanitárias nas etapas produtivas de ante mortem e post mortem com o objetivo de identificar alterações sanitárias na carcaça do animal. Caso algum problema seja identificado, pode ocorrer a condenação parcial ou total da carcaça (PORTILHO, 2016).

A ocorrência da cisticercose bovina pode acarretar em prejuízos financeiros para produtores rurais e indústria de processamento (frigorífico), em virtude do descarte de carcaças condenadas. Sendo assim, atribui-se a importância do desenvolvimento de ações que visem a sanidade do animal, com o objetivo de realizar um controle de patologias que causem tanto riscos à saúde humana, quanto perdas produtivas (FELIPPE et al., 2014).

A cisticercose não apresenta sintomatologia clínica nos animais durante o ciclo produtivo nas propriedades rurais, porém quando identificada no processo de abate nos frigoríficos, a condenação da carcaça pode ser total ou parcial. A infecção dos animais geralmente ocorre na ingestão de água e pastagens que estejam contaminadas com os ovos do parasita (SANTOS et al., 2008).

Diante desse contexto, objetivou-se nesse estudo verificar o quantitativo de condenações totais de carcaças bovinas do estado de Mato Grosso do Sul cuja a causa tenha sido a cisticercose (que engloba as categorias de cisticercose, cisticercose viva e cisticercose calcificada). Além disso, pretende-se determinar a perda econômica dessas condenações e o período de tempo analisado foi de 2013 a 2017. 


\section{MATERIAIS E MÉTODOS}

Foi realizado um estudo retrospectivo sobre as condenações totais de carcaças bovinas, por cisticercose bovina, no estado do Mato Grosso do Sul durante o período de 2013 a 2017, utilizando os registros da base de dados do SIF.

As variáveis selecionadas foram: (i) total de abates de bovinos; (ii) total de condenações totais de carcaças bovinas; (iii) total de carcaças condenadas totalmente por cisticercose; (iiv) total de carcaças condenadas totalmente por cisticercose viva; (v) total de condenações totais por cisticercose calcificada. Vale a pena ressaltar que o total de condenações totais de carcaças bovinas pela causa de cisticercose considerada neste estudo foi alcançado a partir do somatório dos registros de condenações totais por cisticercose, cisticercose viva e cisticercose calcificada.

Para a determinação da perda econômica, foi utilizada a Equação 1.

$$
\text { Perda econômica }_{\text {ano }}=Q C D \times R M C C \times P M A C B
$$

Em que: $Q C D=$ Quantidade de carcaças condenadas totalmente no ano; $\mathrm{RMCC}=$ Rendimento médio de carne por carcaça; e, PMACB= Preço médio anual da arroba (@) de carne bovina. O rendimento médio de carne por carcaça foi de 16 @ e a série de preços foi obtida na base de dados do Agrolink.

A atualização dos valores nominais da perda econômica para o período de 2013 a 2017 foi realizada a partir da Equação 2.

$$
\text { Valor real } l_{\text {ano }}=\left(\frac{\text { Valor nominal }_{\text {ano }}}{I G P-D I_{\text {ano }}}\right) \times I G P-D I_{\text {base }}
$$

Em que: Valor real ano $=$ valor da perda econômica atualizada para o ano de 2017; Valor nominal ano = valor da perda econômica calculada para cada ano do período de 2013 a 2017; IGP-DI ano = Índice Geral de Preços para cada ano do período de 2013 a 2017; e, IGP-DI base = Índice Geral de Preços do ano de 2017.

\section{RESULTADOS E DISCUSSÃO}

\section{ASPECTOS ECONÔMICOS DE CONDENAÇÕES TOTAIS DE CARCAÇAS BOVINAS POR CISTICERCOSE}

No período de 2013 a 2017, no estado de Mato Grosso do Sul (MS) foi realizado um total de 17,10 milhões de abates bovinos, e foram condenados totalmente 56,50 mil carcaças bovinas. Dessas condenações, 15,71 mil foram por cisticercose bovina, representando $27,8 \%$ do total de condenações no período analisado (MAPA, 2018).

$\mathrm{Na}$ Tabela 1 são apresentadas informações sobre 0 total de abates, condenações totais e por cisticercose em carcaças bovinas para as unidades federativas da região Centro-Oeste do país, bem como a perda econômica das carcaças condenadas totalmente por cisticercose. 
TABELA 1. Quantidade de abates, de condenações totais e condenações por cisticercose bovina para os estados que compõem a região Centro-Oeste, bem como a perda econômica para o período de 2013 a 2017

\begin{tabular}{ccccc}
\hline Localidade & $\begin{array}{c}\text { Total de } \\
\text { Abates } \\
\text { (unidades) }\end{array}$ & $\begin{array}{c}\text { Condenações } \\
\text { totais } \\
\text { (unidades) }\end{array}$ & $\begin{array}{c}\text { Condenações totais } \\
\text { por cisticercose } \\
\text { (unidades) }\end{array}$ & $\begin{array}{c}\text { Perda Econômica } \\
\text { por cisticercose } \\
\text { (R\$- reais) }\end{array}$ \\
\hline Mato Grosso & 23.266 .727 & 201.451 & 852 & $1.656 .769,35$ \\
Mato Grosso & $\mathbf{1 7 . 1 0 5 . 1 4 7}$ & $\mathbf{5 6 . 5 0 2}$ & $\mathbf{1 5 . 7 1 7}$ & $\mathbf{3 4 . 2 1 2 . 2 3 7 , 9 4}$ \\
$\begin{array}{c}\text { do Sul } \\
\text { Goiás }\end{array}$ & 14.066 .040 & 36.167 & 17 & $31.563,87$ \\
$\begin{array}{c}\text { Distrito Federal } \\
\text { Total }\end{array}$ & - & - & - & $0,00-$ \\
\hline
\end{tabular}

Fonte: Elaborado a partir de dados do MAPA (2018) e informações do Agrolink (2018) e FGV (2018)

No período de 2013 a 2017, na região Centro-Oeste brasileira foi realizado um total de 54,43 milhões de abates de bovinos. Dos quais, 294,12 mil carcaças foram condenadas totalmente, que corresponde a $0,54 \%$ do total de abates. Em relação aos registros de condenações totais por cisticercose, com um total de 16,58 mil casos, o que equivale a $5,64 \%$ do total de condenações (Tabela 1 ).

A perda econômica determinada para as condenações totais de carcaças bovinas por cisticercose para a região Centro-Oeste foi de $\mathrm{R} \$ 35,90$ milhões, com uma maior participação do estado de Mato Grosso do Sul (R $\$ 34,21$ milhões), seguidos de Mato Grosso ( $\mathrm{R} \$ 1,65$ milhões) e Goiás ( $\mathrm{R} \$ 31,56 \mathrm{mil}$ ) (Tabela 1).

As perdas econômicas possuem relevância, uma vez que poderiam ser evitadas ou minimizadas através de medidas sanitárias eficazes adotadas durante as etapas de produção de carne bovina. O impacto econômico da cisticercose é diluído ao longo da cadeia produtiva da bovinocultura de corte, porém o segmento dos frigoríficos absorve a maior parte desses prejuízos, por atenderem diretamente as imposições do mercado. (ALMEIDA et al., 2017). A cisticercose causa preocupação a produtores e frigoríficos e por ter um potencial zoonótico, é alvo de condenação por parte do serviço de inspeção, acarretando em prejuízos ao setor (ROSSI et al., 2015).

As identificações de carcaças acometidas por cisticercose acarretam em custos aos frigoríficos, pois quando não são condenadas totalmente, podem passar por tratamento para aproveitamento parcial, como o tratamento térmico por calor, frio e a salga, nestes casos há uma desvalorização no valor comercial da carne (ALMEIDA et al., 2017). Souza et al. (2007) acrescentam como prejuízos aos frigoríficos, a elevação do custo de insumos básicos como a mão de obra e energia elétrica, na etapa de retrabalho para o aproveitamento de parte da carcaça condenada com a presença de cisticercose.

O produtor rural também é prejudicado, pois em caso de condenação total ou parcial, o valor é descontado no pagamento realizado ao pecuarista, podendo haver desvalorização de até $65 \%$ do total recebido quando é identificada na carcaça a forma viva da cisticercose (BAVIA et al., 2012).

O estudo de Rossi et al. (2015) aponta que a cisticercose tem atuado como fator limitante no fluxo de exportação brasileira, pois diversos países impõem barreiras não tarifárias quanto a qualidade da carne, diminuindo assim consideravelmente o mercado de exportação. 
Apesar do impacto financeiro e econômico resultante das condenações totais de carcaças bovinas para a cadeia produtiva de bovinocultura de corte das localidades analisadas, a inspeção de carnes é uma medida de prevenção para evitar a ocorrência de casos de teníase na população brasileira. Segundo Santos et. al. (2012), cerca de 50\% da carne comercializada no mercado brasileiro é originária de abates clandestinos, sem qualquer tipo de inspeção oficial, o que torna essa temática relevante como questão de saúde pública.

\section{ASPECTOS SANITÁRIOS E DE SAÚDE PÚBLICA}

O Regulamento de Inspeção Industrial e Sanitário de Produtos de Origem Animal (RIISPOA) estabelece os critérios para condenação total de carcaças bovinas, quando identificadas a presença da cisticercose bovina (BRASIL, 2017). As carcaças serão condenadas totalmente quando for caracterizada infecções intensas de cisticercose bovina, através da identificação de pelo menos oito cistos, vivos ou calcificados, do Cysticercus bovis, que é a forma larval da Taenia saginata, nos locais de eleição examinados na linha de inspeção. Quando não for caracterizada infecção intensa, descartada a parte acometida, a carcaça pode ser direcionada para aproveitamento parcial/condicional (BRASIL, 2017).

Os bovinos são hospedeiros intermediários da Taenia saginata, por apresentarem suas formas larvais nos tecidos, e geralmente adquirem a doença por ingerir alimento contaminado com os ovos do parasito. Já em humanos, a teníase é adquirida através da ingestão de carnes cruas ou malpassada, que apresentem as larvas vivas do parasito (MENEGOTTO et al., 2017).

Conforme verificado por Almeida et al. (2017), patologicamente a cisticercose em humanos vai ter sua importância dependendo da localização do parasito. Quando localizada no Sistema Nervoso Central (SNC), a neurocisticercose se caracteriza como a doença de origem parasitária mais grave. Pois ela gera quadros de convulsões e ataques epiléticos, enquanto quando presentes na musculatura, geralmente não apresentam manifestações clínicas.

$\mathrm{Na}$ bovinocultura de corte, a elevada presença da cisticercose pode ser caracterizada como um indicativo de problemas sanitários, pois a infecção pode ocorrer pela ingestão tanto de água quanto de pastagens contaminadas com ovos do parasito. Dessa forma, atribui-se a importância da adoção de medidas que visem melhorar as condições de saneamento básico em localidades rurais com o objetivo de diminuição da incidência de infecção dos animais (ALMEIDA et al., 2017). $\mathrm{Na}$ Figura 1 é caracterizada a situação de uma musculatura bovina infectada com a presença de um cisticerco vivo.

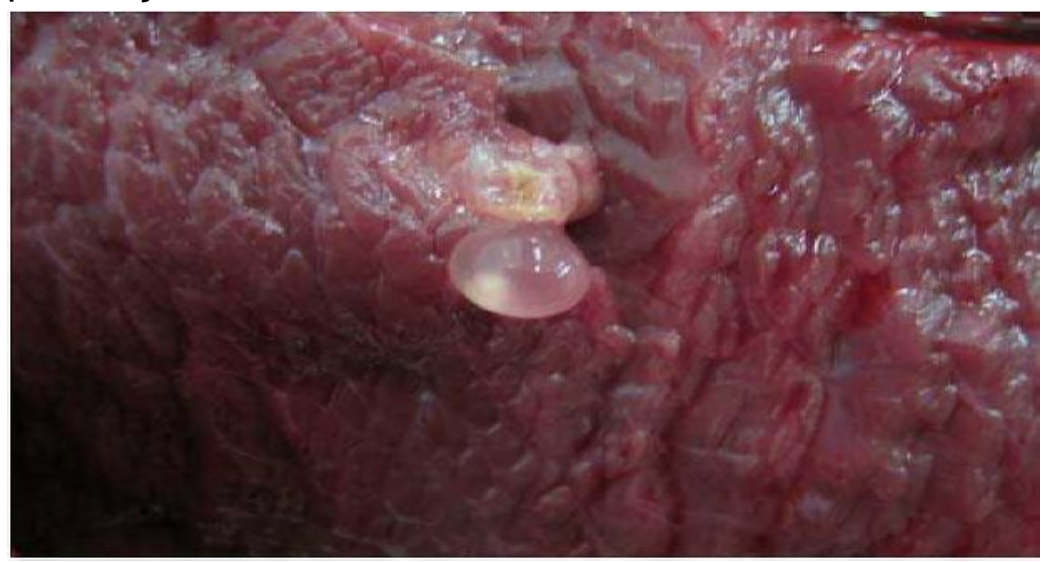

FIGURA 1. Cisticerco vivo na musculatura bovina. Fonte: GOMES (2014, p.8) ENCICLOPÉDIA BIOSFERA, Centro Científico Conhecer - Goiânia, v. 15 n.28; p.128 
Segundo Gomes (2014), apesar da importância da discussão sobre a ocorrência da cisticercose na produção de carne bovina, há certa dificuldade na etapa de diagnóstico clínico definitivo da cisticercose no animal. Então a melhor forma de prevenção para se evitar casos de ocorrência da teníase na população brasileira, é a partir do serviço de inspeção na etapa de abate.

\section{CONCLUSÃO}

A cisticercose é um problema para o setor produtivo de pecuária de corte e também para a saúde pública da sociedade brasileira.

No estado de Mato Grosso do Sul, a perda econômica das condenações totais de carcaças bovinas com a presença de cisticercose determinada foi de $\mathrm{R} \$ 34,12$ milhões para o período de 2013 a 2017. Sendo assim, é necessária a adoção de ações que visem à interrupção do ciclo da cisticercose bovina, como a conscientização dos funcionários de propriedades rurais quanto à importância da doença e sobre a realização do manejo ambiental e do tratamento antiparasitário. Bem como a ampliação do serviço de inspeção sobre a etapa de abate e processamento de carne bovina comercializada no mercado nacional.

\section{REFERÊNCIAS}

AGROLINK. Séries históricas. Disponível em: https://www.agrolink.com.br/cotacoes/historico/ Acesso em: 20 de agosto de 2018.

ALMEIDA, G; LEAL, M. H; SAKAMOTO, C. A. M. Importância econômica e sanitária da cisticercose no Brasil. Revista de Ciência Veterinária e Saúde Pública. UEM, Umuarama, v. 4, Suplem. 2, 2017. Disponível em: http://www.periodicos.uem.br/ojs/index.php/RevCiVet/article/view/39838

BAVIA, M. E; CARNEIRO, D.D.M.T; CARDIM, L.L; SILVA, M.M.N; MARTINS, M.S. Estatística espacial de varredura na detecção de áreas de risco para a cisticercose bovina no estado da Bahia. Arquivo Brasileiro de Medicina Veterinária e Zootecnia, vol.64, n.5, pp.1200-1208, 2012. Disponível em: http://www.scielo.br/pdf/abmvz/v64n5/v64n5a18.pdf

BRASIL. Decreto no 9.013/2017 de março de 2017. RIISPOA - Regulamento de Inspeção Industrial e Sanitária de Produto de Origem Animal. Ministério da Agricultura, Pecuária e Abastecimento e Desenvolvimento Rural, Brasília,2017. Disponível em: http://www.planalto.gov.br/ccivil_03/_Ato20152018/2017/Decreto/D9013.htm

FELIPPE, A. G; PINTO, P.S.A; SANTOS, T.O; NIETO, E.C.A; PEIXOTO, R.P.M.G; SILVA, L.F. Características favoráveis ao controle do complexo teníase-cisticercose em uma região rural de Minas Gerais, Brasil. Revista Brasileira de Ciência $\begin{array}{llllll}\text { Veterinária, } & \text { v. } 21, \quad \text { n. } 4, & \text { p. } 243-246 . & 2014 . & \text { DOI: }\end{array}$ http://dx.doi.org/10.4322/rbcv.2015.303

FGV - FUNDAÇÃO GETÚLIO VARGAS. Indicadores de preços. Disponível em: http://portalibre.fgv.br/main.jsp?lumChannelld=402880811D8E34B9011D92B6B6420 E96 Acesso em: 20 de agosto de 2018.

GOMES, M. A. R. B., Ocorrência da cisticercose bovina em frigorífico localizado na zona da mata mineira. 2014. 35 f. Dissertação (Mestrado Profissional em Zootecnia) - Universidade Federal de Viçosa - UFV. Viçosa, 2014. Disponível em: 
http://www.locus.ufv.br/bitstream/handle/123456789/5981/texto\%20completo.pdf?se quence $=1$

MAPA, Serviço de Inspeção Federal. Quantitativo de abate estadual por Ano/espécie. $2018 . \quad$ Disponível em: http://sigsif.agricultura.gov.br/sigsif_cons/\%21ap_abate_estaduais_cons?p_select=SI $\mathrm{M}$

MENEGOTTO, V. M; BOGO, M. C; SAKAMOTO, C. A. M. Epidemiologia da cisticercose bovina no Estado do Paraná. Revista de Ciência Veterinária e Saúde Pública. UEM, Umuarama, v. 4, Suplem. 2, 2017. Disponível em: http://www.periodicos.uem.br/ojs/index.php/RevCiVet/article/view/39831

PORTILHO, E.F. Análise retrospectiva da implantação do serviço de inspeção municipal de Rio Verde - GO e de microrganismos patogênicos em produtos cárneos. 2016. 42 f. Tese (Doutorado em Medicina Veterinária) - Universidade Estadual Paulista, Faculdade de Ciências Agrárias e Veterinárias. Jaboticabal, 2016. Disponível

em:

https://repositorio.unesp.br/bitstream/handle/11449/134383/portilho_ef_dr_jabo.pdf?s equence $=3$

ROSSI, G. A. M; HOPPE, E.G; MATHIAS, L.A; MARTINS, A.M; MUSSI, L.A; et al. Bovine cysticercosis in slaughtered cattle as an indicator of Good Agricultural Practices (GAP) and epidemiological risk factors. Preventive Veterinary Medicine, v. 118, n. 4, p. 504-508. 2015. DOI: https://doi.org/10.1016/j.prevetmed.2015.01.004 Disponível em: https://www.sciencedirect.com/science/article/pii/S0167587715000057?via\%3Dihub

SANTOS, D.G.N; FINGER, I.R.B; MONÇALVES, V.A; AZEVEDO, M.L Inspeção de carnes: importância na saúde do consumidor. Anais do Salão Internacional de Ensino, Pesquisa e Extensão. v. 4, n. 3, Universidade Federal do Pampa. 2012. Disponível em: http://seer.unipampa.edu.br/index.php/siepe/article/view/209/0

SANTOS, V.C.R., RAMOS, E.T.R., FILHO, F.S.A., PINTO, J.M.S., MUNHOZ, A.D. Prevalência da cisticercose em bovinos abatidos sob Inspeção Federal no município de Jequié, Bahia, Brasil. Revista Ciência Animal Brasileira, v. 9, n. 1, p. 132-139. 2008. Disponível em: https://www.revistas.ufg.br/vet/article/view/766/3437

SOUZA, V.K; PESSÔA-SILVA, M.C; MINOZZO, J.C; THOMAZ-SOCCOL, V. Prevalência da cisticercose bovina no estado do Paraná, sul do Brasil: avaliação de 26.465 bovinos inspecionados no SIF 1710. Semina: Ciências Agrárias, Londrina, v. 28, n. 4, p. 675-684, 2007. Disponível em: http://www.redalyc.org/pdf/4457/445744086014.pdf

TIVERON, D. V. Inspeção pós-morte de bovinos: ocorrência de alterações sanitárias no abate e respectivo impacto em relação ao mercado globalizado. 2014. vi, 56 p. Dissertação (Mestrado em Medicina Veterinária) - Universidade Estadual Paulista Júlio de Mesquita Filho, Faculdade de Ciências Agrárias e Veterinárias de Jaboticabal, $2014 . \quad$ Disponível em: http://hdl.handle.net/11449/110620. 